\title{
FINITE ELEMENT BASED ADAPTIVE NEURO-FUZZY INFERENCE TECHNIQUE FOR PARAMETER IDENTIFICATION OF MULTI-LAYERED TRANSPORTATION STRUCTURES
}

\author{
Kasthurirangan Gopalakrishnan ${ }^{1}$, Siddhartha Kumar Khaitan ${ }^{2}$ \\ ${ }^{1}$ Dept of Civil, Construction and Environmental Engineering, Iowa State University, Ames, USA \\ ${ }^{2}$ Dept of Electrical and Computer Engineering, Iowa State University, Ames, USA \\ E-mails: ${ }^{1}$ rangan@iastate.edu; ${ }^{2}$ skhaitan@iastate.edu
}

Received 21 November 2008; accepted 1 February 2010

\begin{abstract}
During the service life of a pavement, it is often required to conduct Non-destructive tests (NDTs) to evaluate its structural condition and bearing capacity and to detect damage resulting from the repeated traffic and environmental loading. Among several currently used NDT methods, the Falling Weight Deflectometer (FWD) is the most commonly used pavement NDT method applied by many transportation agencies all over the world. Non-destructive testing of pavements using FWD is typically accompanied by the prediction of the Young's modulus of each layer of the pavement structure through an inverse analysis of the acquired FWD deflection data. The predicted pavement layer modulus is both an indicator of the structural condition of the layer as well as a required input for conducting mechanistic-based pavement structural analysis and design. Numerous methodologies have been proposed for backcalculating the mechanical properties of pavement structures from NDT data. This paper discusses the development of an Adaptive-Network-based Fuzzy Inference System (ANFIS) combined with Finite Element Modeling (FEM) for the inverse analysis of the multi-layered flexible pavement structures subjected to dynamic loading.
\end{abstract} element.

Keywords: transportation structures, non-destructive testing, pavement, neural networks, fuzzy inference, finite

\section{Introduction}

Since pavement structures wear down and deteriorate under heavy axle loadings and environmental influences, they need to be maintained and rehabilitated on a regular basis. This requires a very significant commitment of resources on the part of nation's highway agencies at the State, Federal and local levels. For instance, total highway expenditure by all units of the Government was $\$ 126.7$ billion in 2000 which is a 203 percent increase compared to 1980 (average annual increase in 10 percent) (Guide for Mechanistic-Empirical ... 2004). The sheer magnitude of annual expenditures on highway maintenance justifies the application of the best available test procedures and technologies to optimize the use of highway funds.

Various NDT methods have been developed to routinely assess the existing pavement structural condition and subsequently identify the necessary corrective actions. Among them, the Falling Weight Deflectometer (FWD) is the most commonly used NDT device for evaluating the structural state of pavements. FWD can either be mounted in a vehicle or on a trailer and is equipped with a weight and several velocity transducer sensors. To perform a test, the vehicle is stopped and the loading plate (weight) is positioned over the desired location. The sensors are then lowered to the pavement surface and the weight is dropped.

The advantage of an impact load response measuring device over a steady state deflection measuring device is that it is quicker, the impact load can be easily varied and it more accurately simulates the transient loading of moving traffic. Sensors located at specific radial distances monitor deflection history. The deflections measured at radial distances away from the load form the deflection basin. In order to accurately calculate the pavement structural capacity, the deflection basins should also be precisely measured and analyzed. Although there are numerous methods for evaluating the structural capacity of pavements from deflection basin data, there is no standard or universally accepted procedure that presently exists (Layer Moduli Backcalculation ... 1993).

Inverse or back analysis is used to determine the Young's modulus of pavement layers based on the measured deflection data. In this process, more commonly referred to as backcalculation, a numerical optimization 
method is employed so that the measured deflection basin agrees with the deflections given by a numerical model. The optimization process is an iterative method modifying the elastic modulus of the pavement layers until a better adjustment is produced. Moreover, the optimization process can be carried out by employing an algorithm of parameter identification like non-linear least-squares algorithm, research in a database or soft computing methods such as Artificial Neural Networks (ANNs), Genetic Algorithms (GAs) etc. Especially, in recent years, ANNs have been shown to be capable of predicting the pavement layer moduli using the FWD field deflection measurements (Meier and Rix 1995; Ceylan et al. 2007).

The objective of this paper is to investigate the feasibility of using Adaptive-Network-based Fuzzy Inference System (ANFIS) for the inverse analysis of the multi-layered flexible pavement structures based on FWD data. In this approach, a Finite Element (FE) model is employed to envisage the response of the pavement to FWD load with the known characteristics of pavement materials. The FE model captures the non-linear, stressdependent behavior of geo-materials used in the underlying unbound pavement layers resulting in realistic materials characterization and modeling responses.

\section{Non-Destructive Testing of Pavements Using FWD and Interpretation of FWD Data}

FWD equipment measures pavement surface deflections from the applied dynamic load that simulates a moving wheel (Use of Nondestructive ... 2004). There are many advantages to using FWD tests in lieu of or to supplement and traditional destructive tests on structural pavement evaluation. Without FWD testing, structural data must be obtained from numerous cores, borings and excavation pits on the existing highway/ airport pavements. This process can be very disruptive to highway/airport operations. FWD tests are economical to perform and data can be collected at up to 250 locations per day. FWD devices have earned the major role in pavement management. The Strategic Highway Research Program (SHRP) adopted the FWD device as a key piece of equipment for assessing a structural capacity of long-term pavement performance (LTPP) test sections. Under the LTPP program, FWD testing is used at all general pavement studies (GPS) and test sites of specific pavement studies (SPS).

During FWD testing, typically a 9,000-lb load is applied to the pavement surface by the intermediary of a circular plate (with a diameter of 12 in.) and the generated duration of the half-sine pulse is typically 30 ms. It corresponds to loading time produced by a truck moving at 40 to $50 \mathrm{mph}$. The resulting pavement surface deflections are measured using six geophones at the offsets of 0 (D0), 12 in. (D12), 24 in. (D24), 36 in. (D36), 48 in. (D48), and 60 in. (D60) are intervals from the center of the load. The pavement properties are then backcalculated from the observed dynamic response of the pavement surface to an impulse load (the falling weight) through inverse analysis. The backcalculation of pavement layer properties is a very useful pavement design tool to evaluate structural condition for in-service pavements and to characterize the layer properties as inputs into available numerical or analytical programs.

For flexible pavements considered in this study, several pavement layer moduli backcalculation programs have been proposed in literature such as the AREA method (Hoffman and Thompson 1982), ELMOD, MODULUS, WESDEF (Van Cauwelaert et al. 1989), MODCOMP (Irwin and Szenbenyi 1991; Irwin 1994), etc. Researchers have also developed an ANNbased approach to backcalculation after pioneering the application by Meier and Rix (1995).

Flexible pavements are multi-layered structures consisting of visco-elastic bituminous materials (asphalt concrete) that are relatively rigid and unbound geomaterials the non-linear behavior of which is sensitive to the applied pressures. Fig. 1 displays the details of a typical cross-section of flexible pavement structure revealed after trenching (Garg 2002). The use of a subbase layer is optional and is typically combined with the base layer in flexible pavement analysis and design. To overcome limitations associated with using the multilayered elastic theory (MLET) for the analysis of flexible pavements that assumes the pavement layers to be homogenous, isotropic and linear elastic, the Finite Element (FE) modeling approach has been developed and enables the consideration of heterogeneity, non-linearity and orthotropism of the pavement structure.

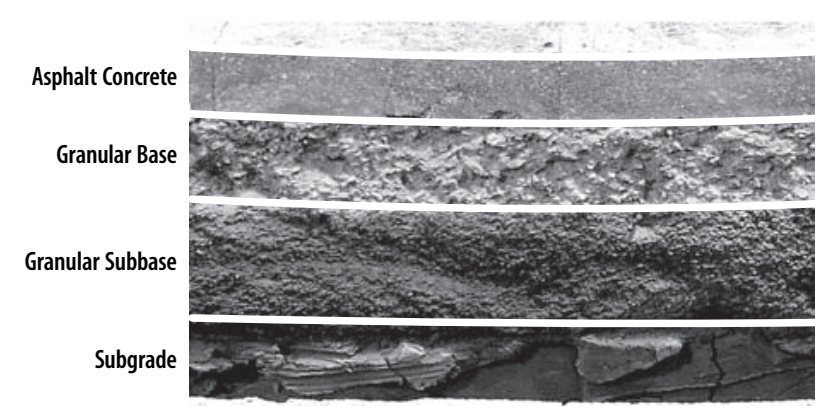

Fig. 1. Typical flexible pavement cross-section after trenching (Garg 2002)

In this paper, an Adaptive-Network-based Fuzzy Inference System (ANFIS) based approach is presented for the backcalculation of the non-linear stiffness properties of the multi-layered flexible pavement structures modeled as 2-D axisymmetric FE structures. Since Jang (1993) proposed ANFIS, its applications are numerous in various fields, including engineering, management, health, biology and even social sciences. Jang et al. (1997) pointed out the following major areas for ANFIS applications: automatic control, pattern recognition, robotics, nonlinear regression, nonlinear system identification and adaptive signal processing. This paper proposes the application of ANFIS for the adaptive backcalculation of pavement layer properties through nonlinear input-output mapping. 


\section{Neuro-Fuzzy Inference Systems Approach}

One of the most important and promising research fields in recent years has been Nature-Inspired Heuristics, an area utilizing some analogies with natural or social systems for deriving non-deterministic heuristic methods to obtain better results in combinatorial optimization problems (Colorni et al. 1996). The fuzzy logic approach (FLA) is one such heuristic method (Zadeh 1965).

In contrast to the classical set theory where the membership of elements is assessed in binary terms (an element either belongs to or does not belong to the set), fuzzy sets are ones the elements of which have membership degrees. The fuzzy set theory permits the gradual assessment of the membership of the elements in a set with the aid of a membership function valued in the real unit interval $[0,1]$.

Fuzzy inference systems (FIS) are powerful tools for the simulation of nonlinear behaviors utilizing fuzzy logic and linguistic fuzzy rules. In literature, there are several inference techniques developed for fuzzy rulebased systems such as Mamdani (Mamdani and Assilian 1975) and Sugeno (Takagi and Sugeno 1985). In the Mamdani fuzzy inference methodology, inputs and outputs are represented by fuzzy relational equations in a canonical rule-based form. In Sugeno FIS, the output of the fuzzy rule is characterized by a crisp function and it was developed to generate fuzzy rules from the given input-output data set. Neuro-fuzzy systems are multilayer feed forward adaptive networks that realize the basic elements and functions of traditional fuzzy logic systems (Oh et al. 2002). Since it has been shown that fuzzy logic systems are universal approximators, neurofuzzy control systems, that are isomorphic to traditional fuzzy logic control systems in terms of their functions, are also universal approximators. ANFIS is an extension of the Sugeno fuzzy model.

The Sugeno model allows fuzzy systems to learn the parameters using the adaptive backpropagation learning algorithm. In general, ANFIS is much more complicated than FIS that can be considered to be a parameterized non-linear map or a crisp function in a consequence called $f$, namely:

$$
f(x)=\frac{\sum_{l=1}^{m} y^{l}\left(\prod_{i=1}^{n} \mu_{A_{i}^{l}}\left(x_{i}\right)\right)}{\sum_{l=1}^{m}\left(\prod_{i=1}^{n} \mu_{A_{i}^{l}}\left(x_{i}\right)\right)},
$$

where: $y^{l}$ is a part of output if Mamdani reasoning is applied or a constant if Sugeno reasoning is applied (Jang et al. 1997). Membership function $\mu_{A_{i}^{l}}\left(x_{i}\right)$ corresponds to input $x=\left[x_{1}, \ldots, x_{n}\right]$ of rule $l$ and $m$ is the number of fuzzy rules. For the $i$-th input predictor variable, $x_{i}$ is real data (for example, the measured FWD deflection) in one point from the set of the observed values. The output values $f(x)$ are the estimated values (for example, back-calculated pavement layer modulus) of the simulation function within the range of an input set (Abolpour et al. 2007). The center of the gravity method is used for defuzzification. This can be further written as:

$$
f(x)=\sum_{l=1}^{m} w_{l} \cdot b_{l}(x),
$$

where: $w_{i}=y^{l}$ and

$$
b_{l}(x)=\frac{\prod_{i=1}^{n} \mu_{A_{i}^{l}}\left(x_{i}\right)}{\sum_{l=1}^{m}\left(\prod_{i=1}^{n} \mu_{A_{i}^{l}}\left(x_{i}\right)\right)} .
$$

If $F_{S}$ is a set of continuous estimated value functions on domain $D$, then $f$ can approximate $F_{S}$ to any desired accuracy. Let $F_{S}$ be a bounded function on $[a, b]$ and $D=\left\{x^{1}, \ldots, x^{h}\right\}-$ a set of points in $[a, b]$. Then there exists the least squares polynomial of degree $\leq r$ between $F_{S}$ and $Q^{h}$ which minimizes the following expression:

$$
\sum_{j=1}^{h}\left|F_{S}\left(x^{j}\right)-Q^{h}\left(x^{j}\right)\right|^{2} .
$$

An overall degree of the polynomial is equal to or less than $r . Q^{h}$ is the real data of output values over $h$-th point of the input set (for each input predictor variable $i=1,2, \ldots, n$ and for each point of real world data $j=$ $1,2, \ldots, h)$.

In Mamdani type of the fuzzy system, real data on the output values can be classified into classes such that the length of each class is equal to $[a, b]$. However, in Sugeno type, the length of $[a, b]$ is only determined over input data set $(D)$ and $f$ can be approximately equal to $F_{S}$; hence, $F_{S}$ is the output values of the simulation model. In the interest of space, the derivation of equations for the development and evaluation of the rule base are not presented in this paper but can be found in Jang et al. (1997). 'Learning' process in ANFIS methodology, namely the adaptation of membership functions to emulate training data, is commonly performed applying two techniques - backpropagation and hybrid learning algorithms. The hybrid optimization method is a combination of Least Squares Error (LSE) and backpropagation descent method. In a hybrid learning algorithm, consequent parameters are identified in forward computation by LSE algorithm and premise parameters are adjusted in backward computation using the backpropagation algorithm.

\section{Parameter Identification of Pavement Systems Using a Neuro-Fuzzy Approach}

Recently, researchers have attempted to employ FIS and ANFIS methodologies to model pavement deflection behavior under dynamic loading (Saltan et al. 2007) and backcalculate the mechanical properties of flexible pavements (Göktepe et al. 2004), respectively. These research studies have shown FLA to be a promising approach for rapid pavement structural evaluation, especially in handling uncertainty and noise associated with field data. In this study, the feasibility of ANFIS methodology for backcalculating non-linear pavement layer moduli from NDT data is further explored.

As the first step towards employing ANFIS methodology in learning the inverse mapping between the 
known input (pavement layer thickness, moduli and Poisson's ratio) and output patterns in a supervised manner, synthetic training and testing databases were generated using a 2-D axisymmetric pavement finite-element software (Raad and Figueroa 1980). The Asphalt Concrete (AC) surface layer was characterized as a linear elastic material. Stress-dependent elastic models along with Mohr-Coulomb failure criteria were applied for the base and subgrade layers. The stress-hardening K- $\theta$ model was used for the base layer:

$$
E_{R}=\frac{\sigma_{D}}{\varepsilon_{R}}=K \cdot \theta^{n}
$$

where: $E_{R}$ is resilient modulus (psi); $\theta$ is bulk stress (psi); $K$ and $n$ are statistical parameters.

The fine-grained low-strength subgrade was modeled using the bi-linear model for characterizing the resilient modulus:

$$
\begin{aligned}
& E_{R}=E_{R i}+K_{1} \cdot\left(\sigma_{d}-\sigma_{d i}\right) \text { for } \sigma_{d}<\sigma_{d i} ; \\
& E_{R}=E_{R i}+K_{2} \cdot\left(\sigma_{d}-\sigma_{d i}\right) \text { for } \sigma_{d}>\sigma_{d i},
\end{aligned}
$$

where: $E_{R}$ is resilient modulus (psi), $\sigma_{d}$ is applied deviator stress (psi) and $K_{1}$ and $K_{2}$ are statistically determined coefficients from laboratory tests. The bi-linear model is a commonly used resilient modulus model for subgrade soils. The value of the resilient modulus at the breakpoint in the bi-linear model $E_{R i}$, can be used to classify fine-grained soils as being soft, medium or stiff. The effect of 9,000 lb FWD impact loading on the flexible pavement structure was simulated in the FE software over typical ranges of AC surface and base layer thicknesses and moduli ranges (see Table).

In developing the ANFIS-based backcalculation approach, input parameters were partitioned using a grid partitioning technique and Gaussian membership functions were used. First order Sugeno FIS with a linear output function was selected as the inference system. ANFIS structure was completed by the selection of a hybrid learning algorithm and a batch learning scheme was used. In this learning algorithm, the backpropagation (BP) algorithm is applied to learning premise parameters while the least square algorithm is applied to learning consequent parameters. In the ANFIS hybrid learning algorithm, the selection of step-size has a great effect on the convergence of the system. The convergence of BP algorithm based on gradient descent will be very slow if the step size is too small. On the other hand, the use of large step size will result in the oscillation of parameter estimation. To overcome this problem, Jang et al. (1997) proposed an intuitive variable-step method used in this study:
1. If the control performance index decreases monotonously in continuous 4 epochs, the step is increased by $10 \%$ to quicken the convergence process.

2. If the control performance index goes through the ascent-descent oscillation in continuous 2 epochs (4 epochs in total), the step is decreased by $10 \%$ to make the convergence process to be more stable.

In the rule base, fuzzy variables were connected with T-norm (fuzzy AND) operators and rules were associated using a max-min decomposition technique. The output part of each rule uses a linear defuzzifier formula; the total output of ANFIS is the weighting average of the output of each rule. The FE-based neuro-fuzzy backcalculation approach was implemented in MATLAB ${ }^{\circ}$ using the in-built toolbox.

Göktepe et al. (2004) used 9 input variables and 1250 training patterns which resulted in an extremely large rule-base and long computing hours. Therefore, they concluded that ANFIS methodology and fuzzy partitioning were not appropriate for a multivariate nonlinear approximation problem comprising 9 input variables. In the same study, Göktepe et al. (2004) employed ANFIS in a scenario involving a considerable amount of uncertainty or having incomplete deflection data and found the ANFIS approach to be successful.

\section{Discussion of Results}

In this research, several case studies were conducted to evaluate the robustness of the finite element based neuro-fuzzy approach developed for pavement layer moduli backcalculation. In the first case study, first four FWD deflections (D0, D12, D24, and D36) along with AC layer thickness $\left(T_{a c}\right)$ and base course thickness $\left(T_{b c}\right)$ were used as inputs (a scenario involving incomplete datasets) and two separate ANFIS models were employed to predict $A C$ modulus $\left(E_{A C}\right)$ and non-linear subgrade modulus $\left(E_{R i}\right)$ (see Fig. 2).

In the first case study, input variables were fuzzified by dividing them into 3 partitions. This paper did not currently focus on backcalculating the base layer moduli due to the associated challenges identified in the previous studies (Meier and Rix 1995). One hundred training patterns from the FE-generated synthetic database were randomly selected as inputs for training in ANFIS and 40 testing vectors were independently selected from the synthetic database to check the prediction ability of the developed ANFIS-based backcalculation model. The range of surface deflections used in the test data set is depicted in Fig. 3. ANFIS methodology predictions for $E_{A C}$ and $E_{R i}$ are shown in Fig. 4 with just 10 epochs.

Table. Ranges of pavement layer properties for generating finite element solutions

\begin{tabular}{lllll}
\hline Material & Layer thickness & Material resilient modulus model & Layer modulus range & Poisson's ratio $(\gamma)$ \\
\hline Asphalt concrete & $3-28$ in. & linear elastic & $100-6000 \mathrm{ksi}$ & 0.35 \\
\hline Unbound aggregate base & $4-22$ in. & non-linear K- $\theta$ model & $K=3-12 \mathrm{ksi}$ & $\begin{array}{l}0.35 \text { for } K \geq 5 \mathrm{ksi} \\
0.40 \text { for } K<5 \mathrm{ksi}\end{array}$ \\
\hline Fine-grained subgrade & $\sim \infty$ & non-linear bi-linear model & $E_{R i}=1-15 \mathrm{ksi}$ & 0.45 \\
\hline
\end{tabular}


a)

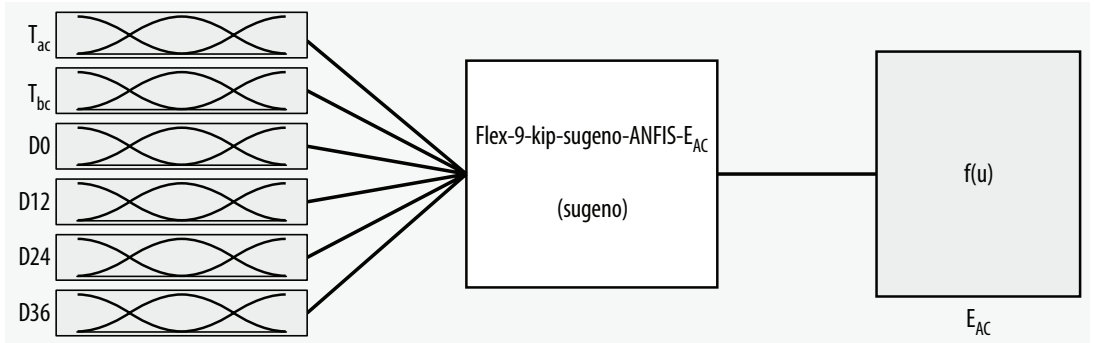

b)

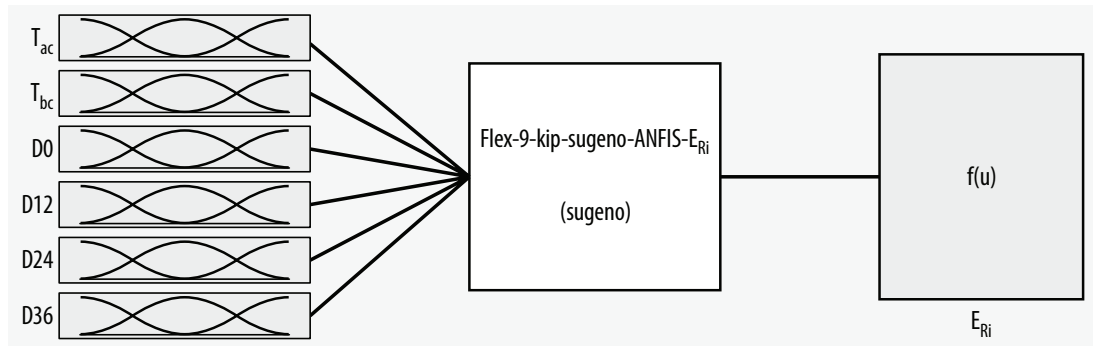

Fig. 2. Case study 1: a - ANFIS model for predicting AC moduli $\left(E_{A C}\right)$; $\mathrm{b}$ - ANFIS model for predicting subgrade moduli $\left(E_{R i}\right)$

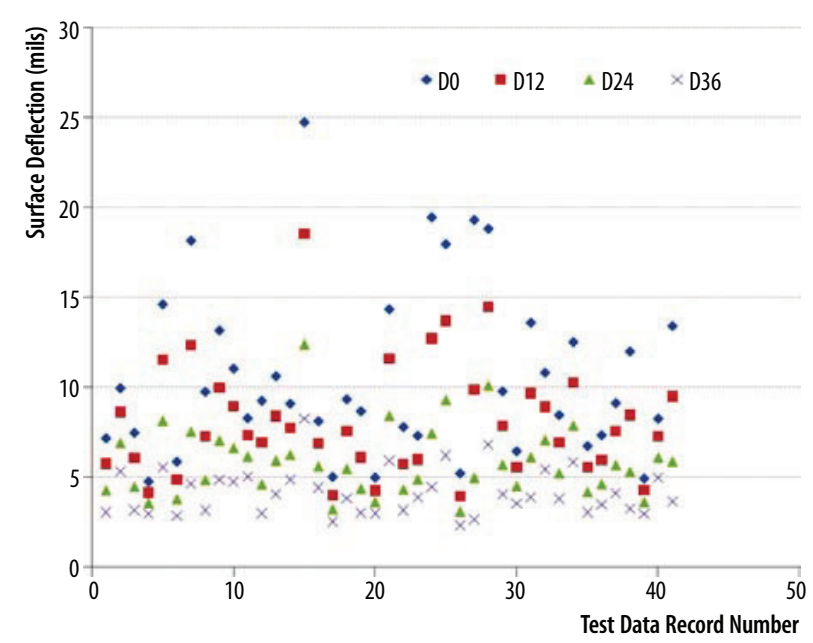

Fig. 3. Case study 1 . Test data surface deflection ranges
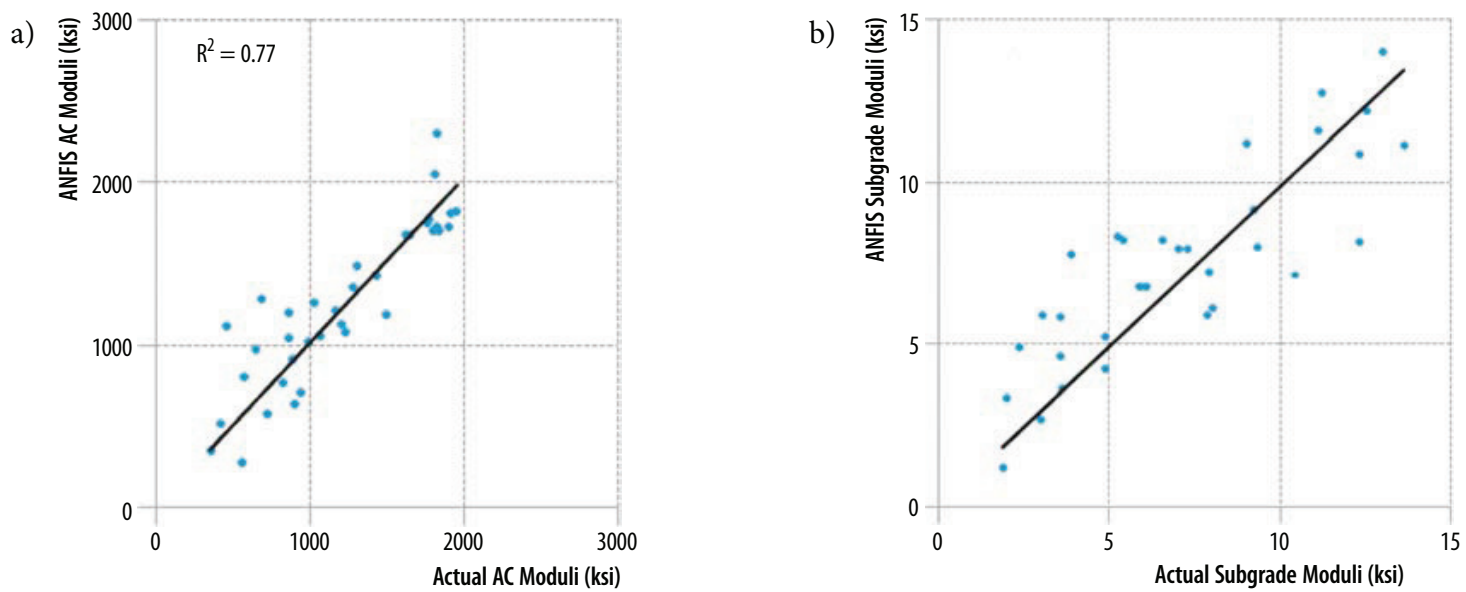

Fig. 4. Case study 1 . ANFIS predictions versus actual moduli 
The Average Absolute Errors (AAEs) were calculated as a sum of individual absolute relative errors divided by the number of independent testing patterns (where $i$ is the $i$ th testing pattern among $n$ testing patterns).

$$
\begin{aligned}
& \text { Average Absolute Error }(A A E), \%= \\
& \sum_{i=1}^{n}\left|\frac{y_{\text {actual }}-y_{\text {predicted }}}{y_{\text {actual }}}\right|_{i} \cdot 100 .
\end{aligned}
$$

The AAEs were reported to be $19 \%$ and $29 \%$ for $E_{A C}$ and $E_{R i}$ respectively which is not acceptable. However, considering the reduced number of deflections used and the smaller training set with fewer epochs, the results demonstrate the neuro-fuzzy backcalculation approach to be promising, especially when uncertainties are involved in the inputs. The surface plots for $E_{A C}$ and $E_{R i}$ with respect to AC surface layer thicknesses and sur- face deflections are shown in Fig. 5. Deflection D36 is especially associated with having influence on subgrade modulus.

In the second case study, ANFIS backcalculation models were developed with all six FWD deflections (D0, D12, D24, D36, D48 and D60) and layer thicknesses as inputs (see Fig. 6). In this case, input variables were fuzzified by dividing them into 2 partitions resulting in two membership functions for each input variable. Five hundred synthetic datasets covering a wide range of pavement layer thicknesses and moduli values were used to train the ANFIS models for predicting $E_{A C}$ and $E_{R i}$ and 100 independent datasets were used for testing the ANFIS models. The surface deflections for the test dataset are shown in Fig. 7. In this case study, three different maximum epochs $(1,5$, and 15) were considered in training the ANFIS backcalculation models.
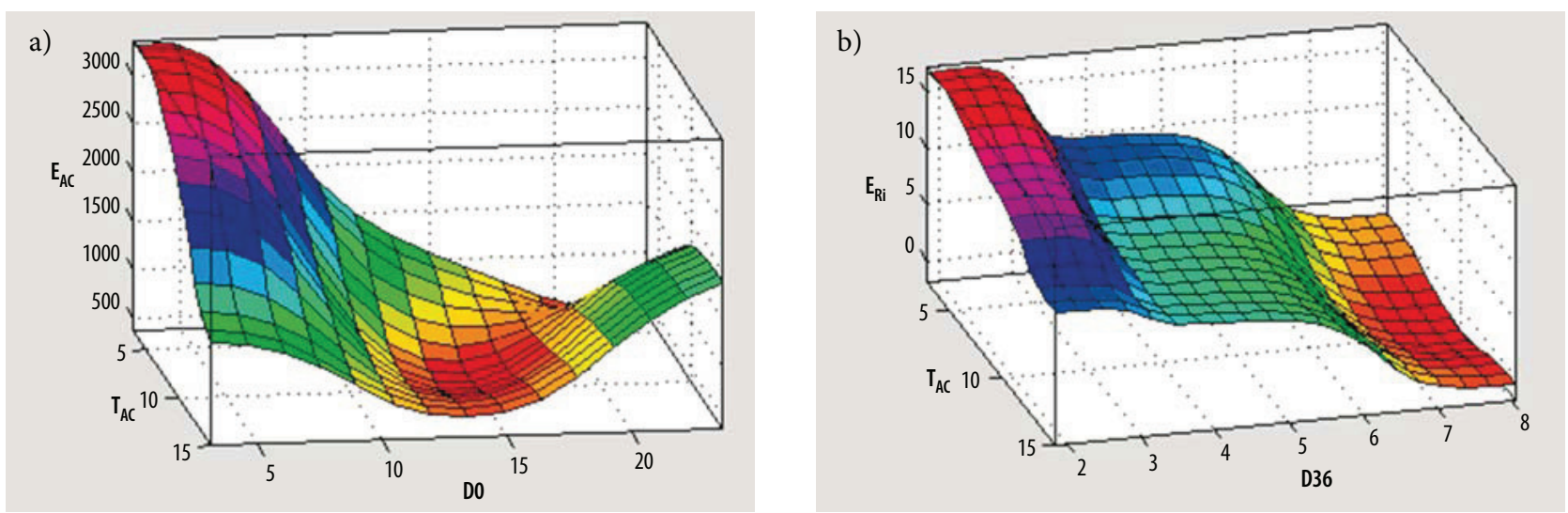

Fig. 5. Case study 1. Surface plots: a - AC moduli $\left(E_{A C}\right) ; \mathrm{b}-\operatorname{subgrade}$ moduli $\left(E_{R i}\right)$

a)

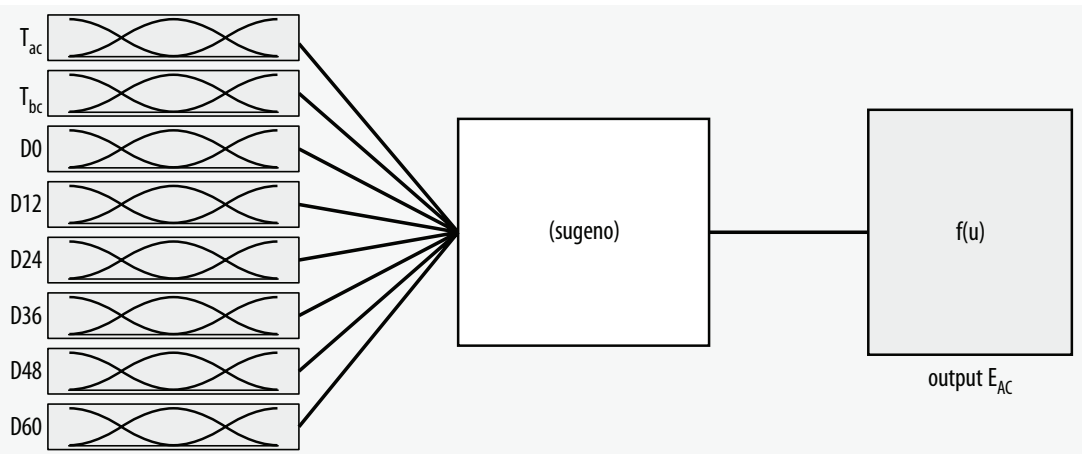

b)

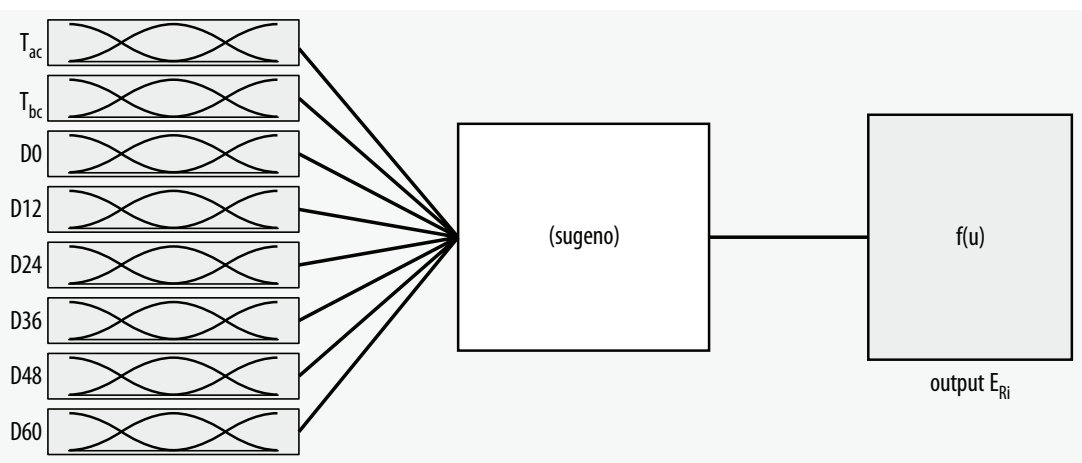

Fig. 6. Case study 2: a - ANFIS model for predicting AC moduli $\left(E_{A C}\right) ; \mathrm{b}$ - ANFIS model for predicting subgrade moduli $\left(E_{R i}\right)$ 


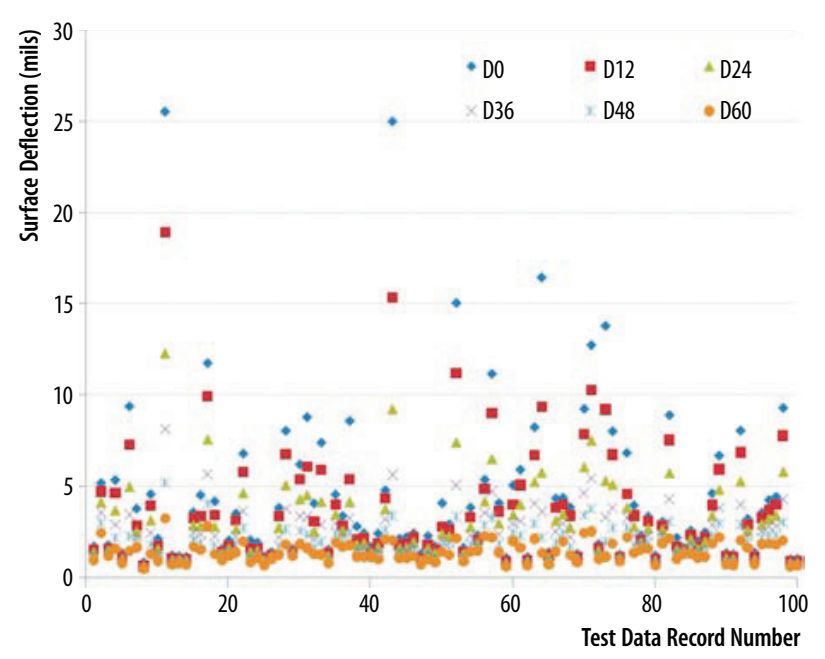

Fig. 7. Case study 2. Test data surface deflection ranges

The ANFIS methodology predictions for $E_{A C}$ and $E_{R i}$ are compared with actual moduli in Figs 8 and 9 respectively. These results demonstrate the feasibility of using the ANFIS based backcalculation approach for the non-linear parameter estimation of the multilayered pavement systems. The AAE values for $E_{A C}$ at 1 , 5 , and 15 epochs were $6.8 \%, 4.7 \%$ and $3.9 \%$ respectively. Similarly, the AAE values for $E_{R i}$ at 1,5, and 15 epochs were $7.2 \%, 1.5 \%$ and $3.7 \%$ respectively. Considering a relatively fewer number of datasets used for training the neuro-fuzzy system and a fewer number of epochs used in achieving very high $R^{2}$ values, it can be concluded that the ANFIS based backcalculation approach is especially suitable where uncertainties are involved in the inputs and it has the adaptive ability to dynamic changes in the environment.

\section{Conclusion}

Various Non-Destructive Test (NDT) methods have been developed to routinely assess the existing pavement structural condition and subsequently identify the necessary corrective actions. Among them, the Falling Weight Deflectometer (FWD) is the most commonly used NDT device for evaluating the structural state of pavements. Non-destructive testing of pavements using FWD is typically accompanied by predicting the Young's modulus of each layer of the pavement structure through backcalculation or the inverse analysis of the acquired FWD deflection data. This paper demonstrated the feasibility of using Adaptive-Network-based Fuzzy Inference System (ANFIS) for the inverse analysis of the multi-layered flexible pavement structures based on FWD data. It was shown that the ANFIS based backcalculation approach inherits the fundamental capability of a fuzzy model to especially deal with nonrandom uncertainties associated with vagueness and imprecision associated with the inverse analysis of transient pavement surface deflection measurements. a)

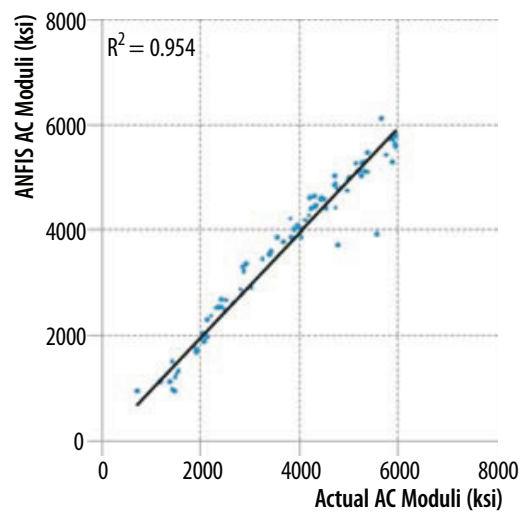

b)

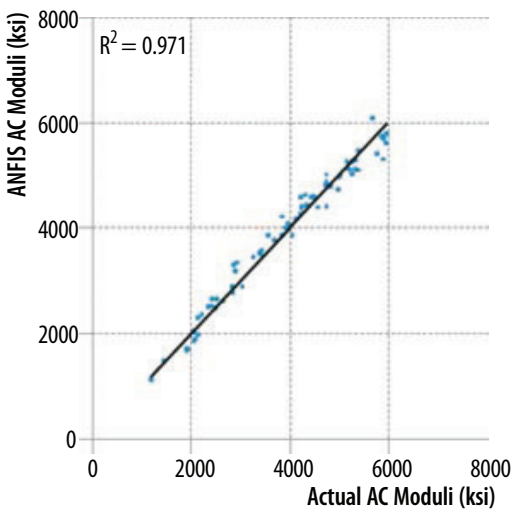

c)

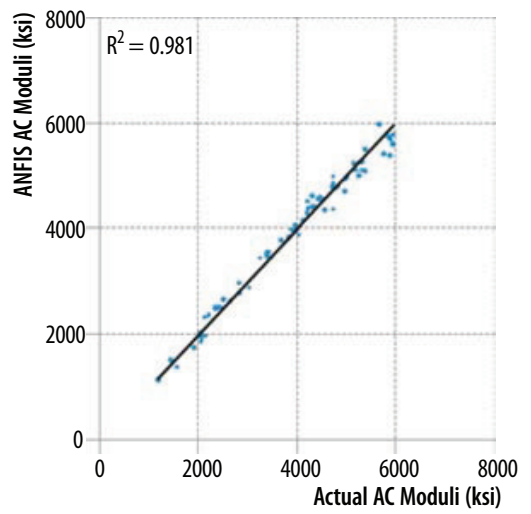

Fig. 8. Case study 2. Comparison of AC moduli: a - 1 epoch; b - 5 epochs; $c-15$ epochs

a)

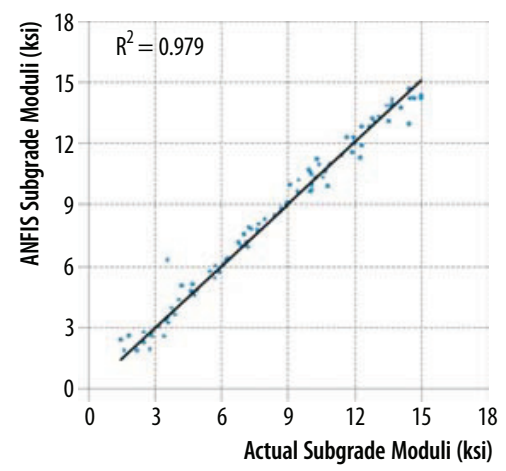

b)

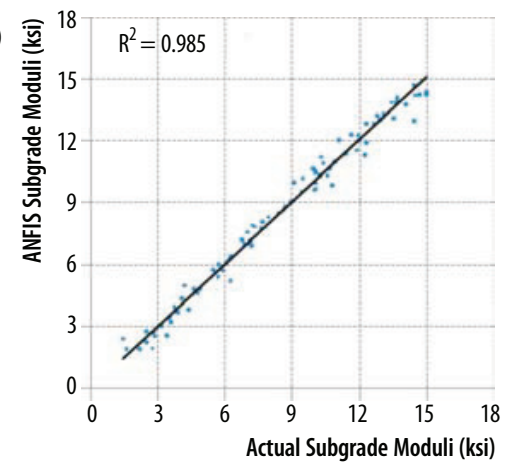

c)

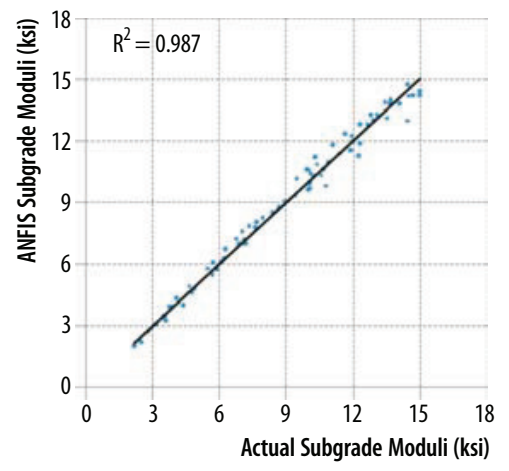

Fig. 9. Case study 2. Comparison of subgrade moduli: $a-1$ epoch; $b-5$ epochs; $c-15$ epochs 


\section{References}

Abolpour, B.; Javan, M.; Karamouz, M. 2007. Water allocation improvement in river basin using adaptive neural fuzzy reinforcement learning approach, Applied Soft Computing 7(1): 265-285. doi:10.1016/j.asoc.2005.02.007

Ceylan, H.; Guclu, A.; Bayrak, M. B.; Gopalakrishnan, K. 2007. Nondestructive Evaluation of Iowa Pavements: Phase I. Final Report. CTRE Project 04-177. Center for Transportation Research and Education (CTRE), Iowa State University, Ames, IA. 136 p. Available from Internet: <www.intrans. iastate.edu/reports/nde-pavements.pdf $>$.

Colorni, A.; Dorigo, M.; Maffioli, F.; Maniezzo, V.; Righini, G.; Trubian, M. 1996. Heuristics from nature for hard combinatorial optimization problems, International Transactions in Operations Research 3(1): 1-21. doi:10.1111/j.1475-3995.1996.tb00032.x

Use of Nondestructive Testing in the Evaluation of Airport Pavements. 2004. U.S. Department of Transportation, Federal Aviation Administration (FAA). FAA Advisory Circular No. 150/5370-11A. Available from Internet: <http:// purl.access.gpo.gov/GPO/LPS111166>.

Garg, N. 2002. Post-Traffic Activities Conducted in CC1 Flexible Pavements: November 2001 - March 2002. Presented at the FAA Airport Pavement Working Group Meeting, Atlantic City, NJ.

Göktepe, A. B.; Ağar, E.; Lav, A. H. 2004. Comparison of multilayer perceptron and adaptive neuro-fuzzy system on backcalculating the mechanical properties of flexible pavements, ARI - The Bulletin of the Istanbul Technical University 54(3): 65-77. Available from Internet: <www.ari.itu. edu.tr/pre_art4/lav.pdf $>$.

Hoffman, M. S.; Thompson, M. R. 1982. Backcalculating nonlinear resilient moduli from deflection data, Transportation Research Record: Journal of the Transportation Research Board 852: 42-51.

Irwin, L. H.; Szenbenyi, T. 1991. User's Guide to MODCOMP3 Version 3.2. CLRP Report No 91-4, Cornell University, Local Roads Program, Ithaca, NY.

Irwin, L. H. 1994. Instructional Guide for Back-Calculation and the Use of MODCOMP. CLRP Publication No 94-10, Cornell University, Local Roads Program, Ithaca, NY.

Jang, J.-S. R. 1993. ANFIS - Adaptive-Network-Based Fuzzy Inference Systems, IEEE Transactions on Systems Man and Cybernetics 23(3): 665-685. doi:10.1109/21.256541

Jang, J.-S. R; Sun, C.-T.; Mizutani, E. 1997. Neuro-Fuzzy and Soft Computing: A Computational Approach to Learning and Machine Intelligence. Prentice-Hall. 614 p.

Mamdani, E. H.; Assilian, S. 1975. An experiment in linguistic synthesis with a fuzzy logic controller, International Journal of Man-Machine Studies 7(1): 1-13. doi:10.1016/S0020-7373(75)80002-2

Meier, R. W.; Rix, G. J. 1995. Backcalculation of flexible pavement moduli from dynamic deflection basins using artificial neural networks, Transportation Research Record: Journal of the Transportation Research Board 1473: 72-81.

Guide for Mechanistic-Empirical Design of New and Rehabilitated Pavement Structures. 2004. National Cooperative Highway Research Program (NCHRP). NCHRP 1-37A, Final Report

Oh, S.-K.; Pedrycz, W.; Ahn, T.-C. 2002. Self-organizing neural networks with fuzzy polynomial neurons, Applied Soft Computing 2(1): 1-10. doi:10.1016/S1568-4946(02)00023-6

Layer Moduli Backcalculation Procedure: Software Selection. 1993. Final Report. SHRP-P651. Strategic Highway Re- search Program (SHRP), National Academy of Sciences, Washington, D.C. Available from Internet: <http://onlinepubs.trb.org/onlinepubs/shrp/SHRP-P-651.pdf>.

Raad, L.; Figueroa, J. L. 1980. Load response of transportation support systems, Transportation Engineering Journal of ASCE 106(1): 111-128.

Saltan, M.; Saltan, S.; Sahiner, A. 2007. Fuzzy logic modeling of deflection behavior against dynamic loading in flexible pavements, Construction and Building Materials 21(7): 1406-1414. doi:10.1016/j.conbuildmat.2006.07.004

Takagi, T.; Sugeno, M. 1985. Fuzzy identification of systems and its applications, IEEE Transactions on Systems Man and Cybernetics 15(1): 116-132.

Van Cauwelaert, F. J.; Alexander, D. R.; White, T. D.; Barker, W. R. 1989. Multilayer elastic program for backcalculating layer moduli in pavement evaluation, in Nondestructive Testing of Pavements and Backcalculation of Moduli, ASTM STP 1026, A. J. Bush III and G.Y. Baladi, Eds., American Society for Testing and Materials, Philadelphia, 171-188.

Zadeh, L. A. 1965. Fuzzy sets, Information and Control 8(3): 338-353. doi:10.1016/S0019-9958(65)90241-X 